LA-UR-99-5914

\title{
Melting as a String-Mediated Phase Transition
}

\author{
Leonid Burakovsky*, Dean L. Preston† and Richard R. Silbar ${ }^{\ddagger}$ \\ Los Alamos National Laboratory \\ Los Alamos, NM 87545, USA
}

\begin{abstract}
We present a theory of the melting of elemental solids as a dislocation-mediated phase transition. We model dislocations near melt as non-interacting closed strings on a lattice. In this framework we derive simple expressions for the melting temperature and latent heat of fusion that depend on the dislocation density at melt. We use experimental data for more than half the elements in the Periodic Table to determine the dislocation density from both relations. Melting temperatures yield a dislocation density of $(0.61 \pm 0.20) b^{-2}$, in good agreement with the density obtained from latent heats, $(0.66 \pm 0.11) b^{-2}$, where $b$ is the length of the smallest perfect-dislocation Burgers vector. Melting corresponds to the situation where, on average, half of the atoms are within a dislocation core.
\end{abstract}

Key words: melting, dislocation, phase transition, lattice

PACS: 11.10.Lm, 11.27.+d, 61.72.Bb, 64.70.Dv, 64.90.+b

\section{Introduction}

Nearly 50 years ago Shockley [1] successfully accounted for the fluidity of a liquid by assuming a certain concentration of line defects in the liquid state. Bragg [2] had earlier estimated an upper bound on the core energy of a dislocation under the assumption

\footnotetext{
*E-mail: BURAKOV@T5.LANL.GOV

${ }^{\dagger}$ E-mail: DEAN@LANL.GOV

${ }^{\ddagger}$ E-mail: SILBAR@WHISTLESOFT.COM. Also at WhistleSoft, Inc., 168 Dos Brazos, Los Alamos, NM 87544, USA
} 
that the core atomic configuration was like that of a liquid. Cotterill and Doyama [3] later confirmed the Bragg estimate. These early results implied that the liquid state is equivalent to a crystal saturated with dislocation cores. It was first suggested by Mott [4] that the melting transition could be described in terms of dislocations.

There are now compelling results from molecular dynamics [5] and Monte Carlo [6] simulations that imply that dislocations play a key role in three-dimensional melting, and moreover there is experimental evidence that linear defects are in fact generated near the melting transition [7].

Mizushima [8] and Ookawa [9] were the first to formulate a dislocation theory of melting. They based their theory on the fact that the self-energy of a dislocation decreases with dislocation density because of screening. In their theory melting is a first-order transition that occurs when the free energy of the crystal with a sufficiently high concentration of thermally-generated dislocations equals the free energy of the dislocation-free crystal. Their predicted melting temperatures agree with data for reasonable choices of the core energy. Many dislocation theories of melting then followed [10-13], most notably the exhaustive treatment of linear-defect-mediated melting by Kleinert and collaborators [14]. We refer the reader to several fine reviews of the literature for additional details and references on dislocation-mediated melting [15, 16].

Significant progress in our understanding of melting has been achieved by Kleinert 14] who pointed out that the melting process cannot proceed through the mediation of dislocations alone. Dislocations are associated with the discrete translational symmetry of the crystal, so only this symmetry is lost when dislocations condense. But the rotational order of the solid is also lost as the solid converts into liquid, and for this to occur the defects associated with the rotational symmetry of the lattice, namely disclinations, must come into play. Kleinert assumes that the free energy of dislocations alone would lead to a second-order phase transition, and in addition shows that a second-order proliferation of disclinations can occur in a background of dislocations above some critical density. The coupled dislocation-disclination system could, however, undergo a first-order transition, i.e., melting.

Although disclinations must participate in the melting process, in this paper we consider only the dislocation degrees of freedom. Ideally, we would derive the precise form of the free energy of a dense ensemble of dislocations interacting via the full Blin potential 14] and subject to specific configurational constraints (Brownian, self-avoiding, etc.), but this problem has so far defied solution. Instead we develop an effective theory of melting based on perfectly screened, non-interacting dislocations. We employ the widely accepted $-\rho \ln \rho$ form ( $\rho$ is dislocation density) for the self-energy density of dislocations [8, 17, 18], which results in a first-order phase transition. A dislocation in a dense ensemble of other dislocations is assumed to be a random loop, i.e., the possible configurations of a dislocation loop are closed random walks, and short-range steric interactions are neglected. Thus the partition function is evaluated in the independent-loop approximation. We obtain two new relations: a simple expression for the melting temperature (the melting relation) that explicitly takes into account the crystal structure, and another relation between melting temperature, latent heat of fusion, and critical density of dislocations. We carry out a comprehensive comparison of these relations with experimental data on over half of the 
Periodic Table. The melting relation is accurate to $17 \%$ [19]. Dislocation densities as determined from the melting temperature and latent heat relations are $\rho=(0.61 \pm 0.20) b^{-2}$ and $(0.66 \pm 0.11) b^{-2}$, respectively, where $b$ is the length of the smallest perfect-dislocation Burgers vector. Both relations should also apply to alloys and compounds.

In Section 2 we discuss the statistical mechanics of dislocation loops on a lattice. These results are used to derive the melting relation in Section 3, the free energy density in Section 4, and the formula for the latent heat of fusion in Section 5. The values for the critical dislocation density extracted from both the melting relation and the formula for the latent heat of fusion are checked in Section 6 with the formula for volume change at melt. Our concluding remarks appear in Section 7.

\section{Statistical mechanics of dislocation loops on a lat- tice}

The energy per unit length, $\sigma$, of a dislocation can be very large. However, this energy can always be compensated at sufficiently high temperatures by the large entropy of line-like structures, as will be seen in what follows.

In a Bravais lattice with coordination number $z$ we consider the graph $\Gamma$, the edges of which are all nearest-neighbor links. The set of $z$ links from any lattice site is identical to the set of shortest perfect-dislocation Burgers vectors, of length $b$. We now evaluate the partition function for a single Brownian loop on $\Gamma$.

The line tension, $\sigma$, is assumed to be independent of its length, $L$, as is the case for a dislocation in a dense complex network. (In a dilute network, interactions between distant segments of a dislocation lead to a logarithmic dependence of $\sigma$ on $L$.) The number of configurations of a string of length $L$ is $\left(z^{\prime}\right)^{L / b}$, where $z^{\prime}$ is the number of possible directions that a line segment can take from a given lattice site. If backtracking is not allowed, $z^{\prime}=z-1$. For a simple cubic lattice in $D$ dimensions $z=2 D$.

Hence, the partition function for a single closed dislocation (in 3 dimensions) is

$$
Z_{1}=\sum_{L} p(L, V)\left(z^{\prime}\right)^{L / b} e^{-\beta \sigma L}=\sum_{L} p(L, V) e^{-\beta \sigma_{\mathrm{eff}} L}, \quad \sigma_{\mathrm{eff}} \equiv \sigma\left(1-\frac{T \ln z^{\prime}}{\sigma b}\right),
$$

where $\beta \equiv 1 / k_{B} T, p(L, V)$ is the sum of probabilities over all lattice sites that a dislocation of length $L$ will close, $V$ is the volume of the system, and $\sigma_{\text {eff }}$ is the effective energy cost to create unit length of a string at temperature $T$.

In order to calculate $p(L, V)$, let $p\left(\mathbf{r}^{\prime}, \mathbf{r} ; L / b\right)$ be the probability density for a dislocation of $L / b=n$ steps to start at $\mathbf{r}$ and end at $\mathbf{r}^{\prime}$. In the limit $n \rightarrow \infty, b \rightarrow 0$, and $L=$ const, $p\left(\mathbf{r}^{\prime}, \mathbf{r} ; L / b\right)$ satisfies the diffusion equation 20

$$
\frac{\partial p}{\partial n}=\frac{b^{2}}{z^{\prime}} \nabla^{2} p
$$

the solution of which is the heat-kernel expansion

$$
p\left(\mathbf{r}^{\prime}, \mathbf{r} ; L / b\right) \equiv p\left(\mathbf{r}^{\prime}-\mathbf{r} ; L / b\right)=\sum_{\mathbf{k}} f_{\mathbf{k}}(\mathbf{r}) f_{\mathbf{k}}^{*}\left(\mathbf{r}^{\prime}\right) e^{-E_{\mathbf{k}} L / b},
$$


where the $f_{\mathbf{k}}(\mathbf{r})$ are eigenfunctions of the Laplacian,

$$
-\frac{b^{2}}{z^{\prime}} \nabla^{2} f_{\mathbf{k}}=E_{\mathbf{k}} f_{\mathbf{k}}
$$

which we take as normalized according to

$$
\int d^{3} \mathbf{r}\left|f_{\mathbf{k}}(\mathbf{r})\right|^{2}=1
$$

When $V \rightarrow \infty$, we have

$$
f_{\mathbf{k}}(\mathbf{r})=\frac{1}{\sqrt{V}} e^{i \mathbf{k} \cdot \mathbf{r}}, \quad E_{\mathbf{k}}=\frac{b^{2} k^{2}}{z^{\prime}},
$$

where $0 \leq k=|\mathbf{k}| \leq \infty$. It then follows from Eq. (3), in which we replace a sum by an integral, $\sum_{\mathbf{k}} \rightarrow V /(2 \pi)^{3} \int d^{3} \mathbf{k}$, that

$$
p\left(\mathbf{r}^{\prime}-\mathbf{r} ; L / b\right)=\int \frac{d^{3} \mathbf{k}}{(2 \pi)^{3}} e^{i \mathbf{k} \cdot\left(\mathbf{r}-\mathbf{r}^{\prime}\right)-b L k^{2} / z^{\prime}}=\left(\frac{z^{\prime}}{4 \pi b L}\right)^{3 / 2} e^{-z^{\prime}\left(\mathbf{r}^{\prime}-\mathbf{r}\right)^{2} / 4 b L} .
$$

The normalization (5) of the eigenfunctions thus imparts unit normalization to the probability density:

$$
\int d^{3} \mathbf{u} p(\mathbf{u} ; L / b)=1
$$

The partition function for a dislocation loop $\left(\mathbf{r}^{\prime}=\mathbf{r}\right)$ is therefore

$$
Z_{1}=\sum_{L} \frac{b}{L} \int d^{3} \mathbf{r} p(\mathbf{0} ; L / b) e^{-\beta \sigma_{\mathrm{eff}} L}=\left(\frac{z^{\prime}}{4 \pi}\right)^{3 / 2} \frac{V}{b^{3}} \sum_{L}\left(\frac{L}{b}\right)^{-5 / 2} e^{-\beta \sigma_{\mathrm{eff}} L} \equiv \sum_{L} N(L) e^{-\beta \sigma L}
$$

where the factor $b / L$ removes the overcounting due to the degeneracy in the number of starting points on the loop. Here, $N(L)$ is the number of configurations of a loop of length $L$. The exponent $5 / 2$ becomes $1+D / 2$ in $D$ dimensions [21.

Real dislocations are not necessarily Brownian loops. In fact, they are expected to be self-avoiding and/or neighbor-avoiding loops, so they do not penetrate each other's core. Eq. (9) can then be extended to non-Brownian or open dislocations by means of an effective exponent $q+1 \neq 5 / 2$ and normalization constant $A\left(q, z^{\prime}\right)$ [22], as follows:

$$
Z_{1}=A\left(q, z^{\prime}\right) \frac{V}{b^{3}} \sum_{L}\left(\frac{L}{b}\right)^{-q-1} e^{-\beta \sigma_{\mathrm{eff}} L}
$$

Here, $q=-1$ for non-interacting (Brownian) open dislocations 222 and $q \approx 7 / 4$ for selfavoiding dislocations at low densities in 3 dimensions [22]. In the string literature, the value $q=0$ has also been quoted. A general argument based on modular invariance [23] shows that for non-interacting closed strings $q=0$ for sufficiently high energy on any compact target space. The same value of $q$ was also obtained in a discrete model for strings [24], and as a static solution to the string Boltzmann equation [25]. In principle, $q$ may even be a function of temperature. Although we may expect $3 / 2 \leq q \leq 7 / 4$ [12], our 
main conclusions do not depend on the precise value of $q$. The normalization constant, $A\left(q, z^{\prime}\right)$, can be calculated analytically for Brownian loops in any dimension $(q=D / 2)$, analogously to the calculation of $A\left(3 / 2, z^{\prime}\right)=\left(z^{\prime} / 4 \pi\right)^{3 / 2}$ in Eq. (9), and numerically in other cases.

The average length of a loop is

$$
\langle L\rangle=\frac{\sum_{L} L N(L) e^{-\beta \sigma L}}{\sum_{L} N(L) e^{-\beta \sigma L}}=\frac{\xi(T)}{\bar{\xi}(T)} b
$$

where we define

$$
\xi(T) \equiv A\left(q, z^{\prime}\right) \sum_{L / b}\left(\frac{L}{b}\right)^{-q} e^{-\beta \sigma_{\mathrm{eff}} L}
$$

and

$$
\bar{\xi}(T) \equiv A\left(q, z^{\prime}\right) \sum_{L / b}\left(\frac{L}{b}\right)^{-q-1} e^{-\beta \sigma_{\text {eff }} L}=\frac{b^{3}}{V} Z_{1} .
$$

The grand canonical partition function for an ensemble of non-interacting indistinguishable loops is given by

$$
Z=Z(T, V, \mu)=\sum_{N=1}^{\infty} \frac{Z_{1}^{N}}{N !} e^{\mu N / k_{B} T}=\exp \left\{\exp \left(\frac{\mu}{k_{B} T}\right) Z_{1}\right\}
$$

where $\mu$ is the chemical potential. The free energy of the ensemble is

$$
F=-k_{B} T \ln Z=-k_{B} T e^{\mu / k_{B} T} Z_{1} .
$$

The average number of loops in the ensemble is

$$
\bar{N}=-\left(\frac{\partial F}{\partial \mu}\right)_{T, V}=e^{\mu / K_{B} T} Z_{1}
$$

Since $Z=\sum_{L_{i}} N\left(L_{i}\right) e^{-\beta\left(\sigma L_{i}-\mu\right)}$, where $N\left(L_{i}\right)$ is the number of dislocation configurations of total length $L_{i}$, the average total dislocation length in the ensemble is

$$
\begin{gathered}
\bar{L}=\frac{1}{Z} \sum_{L_{i}} L_{i} N\left(L_{i}\right) e^{-\beta\left(\sigma L_{i}-\mu\right)}=-\frac{\partial \ln Z}{\partial(\beta \sigma)}=-e^{\mu / k_{B} T} \frac{\partial Z_{1}}{\partial(\beta \sigma)} \\
=\bar{N} \frac{\sum_{L} L N(L) e^{-\beta \sigma L}}{\sum_{L} N(L) e^{-\beta \sigma L}}=\bar{N}\langle L\rangle,
\end{gathered}
$$

i.e., the average total dislocation length is equal to the average number of loops times the average loop length.

The dislocation density, $\rho$, is the average total length per unit volume. It then follows from (12),(13) and (17) that

$$
b^{2} \rho(T)=\frac{\bar{N}\langle L\rangle}{V} b^{2}=\frac{\bar{N}}{V} \frac{\xi(T)}{\bar{\xi}(T)} b^{3}=e^{\mu / k_{B} T} \xi(T) .
$$




\section{New melting relation}

The effective line tension, $\sigma_{\text {eff }}$, [see Eq. (1)] vanishes at the critical temperature $k_{B} T_{c r} \equiv$ $\sigma b / \ln z^{\prime}$. Consequently, dislocations proliferate as $T_{c r}$ is approached from below. At temperatures above $T_{c r}$, the divergence of $Z_{1}$ signals the breakdown of the underlying theory, and the system enters a new phase. Hence, the temperature $T_{c r}$ corresponds to a phase transition, in which dislocations are copiously produced in the solid. We therefore equate the melting temperature, $T_{m}$, to $T_{c r}$.

The line tension, i.e., the dislocation self-energy per unit length, is assumed to be that of a dislocation in a complex array, or tangle, of other dislocations. In that case the stress field of a given dislocation beyond $\simeq R / 2$, where $R$ is the mean interdislocation separation, is largely cancelled out by the stress fields of the other dislocations in the complex array 26, 27]. The line tension is then the sum of the core energy plus the elastic energy inside a cylinder of radius $R / 2$ [26]:

$$
\sigma=\kappa \frac{G b^{2}}{4 \pi} \ln \left(\frac{\alpha}{b} \frac{R}{2}\right)=\kappa \frac{G b^{2}}{8 \pi} \ln \left(\frac{\alpha^{2}}{4 b^{2} \rho}\right) .
$$

Here, $\kappa$ is 1 for a screw dislocation and $(1-\nu)^{-1} \approx 3 / 2$ for an edge dislocation, $\nu$ being the Poisson ratio. Also, $G$ is the shear modulus, $b$ is the Burgers vector magnitude, and $\alpha$ is a constant of order unity. In the second half of this equation we have taken distance $R$ to be approximately equal to $1 / \sqrt{\rho}$, where $\rho$ is the dislocation density defined in Eq. (18). An expression of the form (19) with $R=\rho^{-1 / 2}$ for the dislocation self-energy was originally proposed by Mizushima [8], later put on a sound theoretical basis by Yamamoto and Izuyama [17], and was recently employed by Kierfeld and Vinokur [18 to model dislocation-mediated phase transitions of vortex-line lattices in high- $T_{c}$ superconductors.

The constant $\alpha$ accounts for the nonlinear elastic effects in the dislocation core. Hirth and Lothe [26] compare dislocation energies in the Peierls-Nabarro (discrete) and Volterra (continuum) dislocation models and find

$$
\frac{1}{\alpha}=\frac{d}{e b}\left(\frac{\sin ^{2} \beta}{e^{\gamma}(1-\nu)}+\cos ^{2} \beta\right),
$$

where $\gamma=(1-2 \nu) / 4(1-\nu) \approx 1 / 8, d$ is the interplanar spacing, and $\beta$ is the angle between the Burgers and sense vectors of the dislocation. In a face-centered cubic (fcc) crystal, the smallest perfect-dislocation Burgers vectors are $\frac{1}{2}\langle 110\rangle a$, and the primary glide planes are $\{111\}$ with $d=a / \sqrt{3}$, where $a$ is the lattice constant. Experimental evidence (ref. [26], Table 9-2, p. 275) suggests that the predominant high-temperature glide system in body-centered cubic (bcc) lattices is $\{110\}$, which has $d=a / \sqrt{2}$. The smallest bcc perfect-

dislocation Burgers vectors are $\frac{1}{2}\langle 111\rangle a$. Thus, in both cases $d / b=\sqrt{2 / 3}$. Averaging over $\beta$, we find $\alpha \approx 2.9$ for both fcc and bcc lattices. Atomistic calculations of core energies in ionic crystals (ref. [26], p. 232) indicate that $\alpha \approx 3$. In metals, no such calculations have been performed. We use $\alpha=2.9$ for all elements.

We have also assumed that no backtracking is allowed for dislocations, $z^{\prime}=z-1$, since each backtracking would result in a divergence in the linear elastic interaction energy 
between the overlapping segments. The coordination numbers for the elements considered in our analysis below are $z=6$ for a simple cubic (sc) lattice, $z=8$ for bcc and bodycentered tetragonal (bct) lattices, and $z=12$ for fcc, hexagonal close-packed (hcp), and double hcp (dhcp) lattices. Replacing

$$
b^{3} \equiv \lambda v_{W S}
$$

where $v_{W S}$ is the volume of the Wigner-Seitz cell of the crystal lattice and $\lambda$ is a geometric constant, we finally obtain our formula for the melting temperature of the elements:

$$
T_{m}=\frac{\lambda G v_{W S}}{4 \pi \delta \ln (z-1)}, \quad \delta^{-1} \equiv \kappa \ln \left(\frac{1.45}{b \sqrt{\rho\left(T_{m}\right)}}\right) .
$$

In ref. [19 we evaluated $G v_{W S} / 4 \pi T_{m} \ln (z-1)$ for 51 elements and found $\delta / \lambda$ to be $1.01 \pm 0.17$, where the error is the root-mean-square deviation. These $\delta / \lambda$ are summarized in Fig. 1.

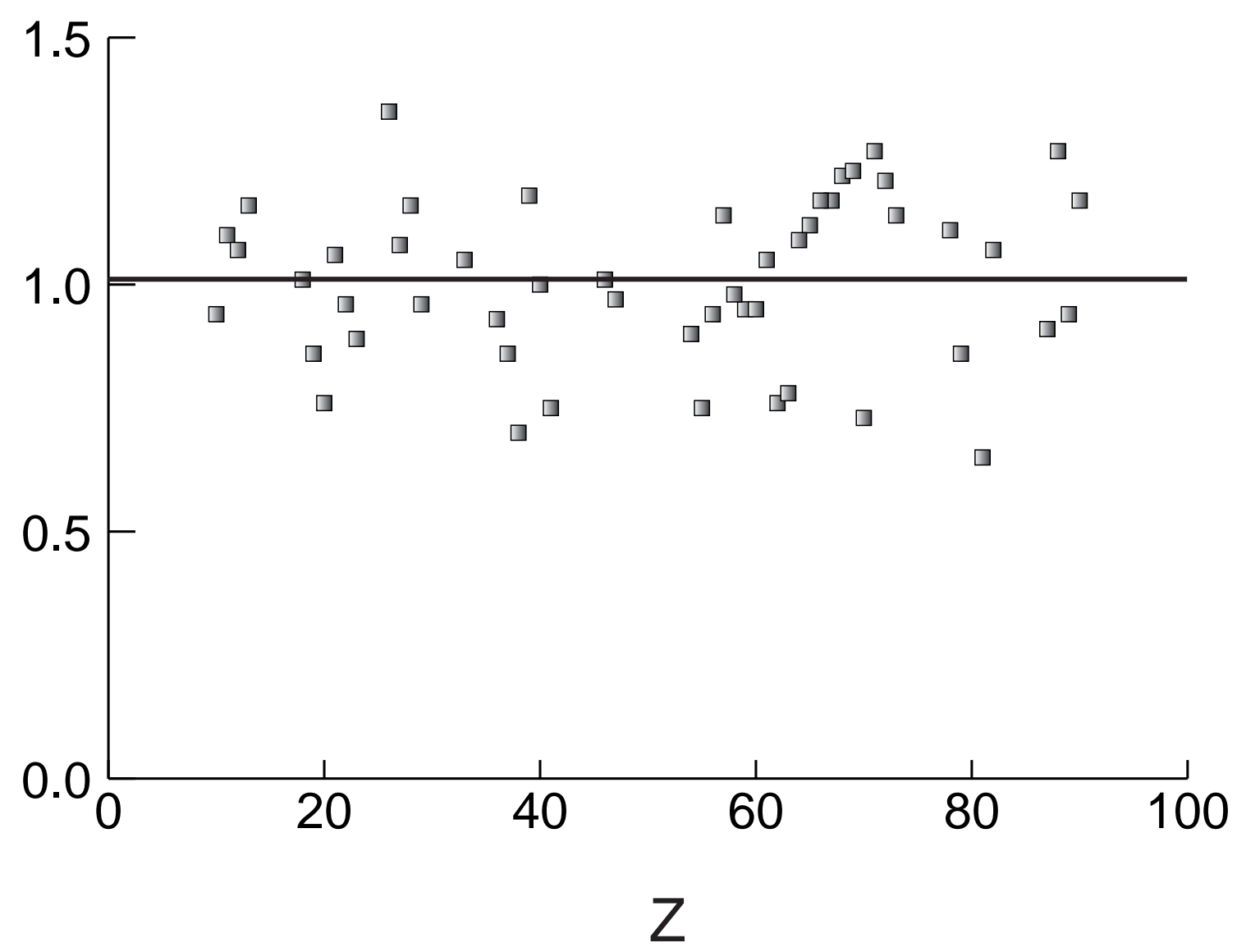

Fig. 1. Values of $\delta / \lambda=G v_{W S} / 4 \pi T_{m} \ln (z-1)$ from experimental data for 51 elements. 
We now assume that the dislocation ensemble is dominated by perfect dislocations with the smallest possible Burgers vectors, since dislocation energy is proportional to $b^{2}$. For a bcc crystal $b=a \sqrt{3} / 2, v_{W S}=a^{3} / 2$, and $b=a / \sqrt{2}, v_{W S}=a^{3} / 4$ for a fcc crystal. Then, $b^{3} \approx 1.30 v_{W S}$ and $1.41 v_{W S}$, respectively. For a hcp lattice, $\lambda=(4 / \sqrt{3})(c / a)^{-1}$, so that for an ideal hcp crystal $(c / a=\sqrt{8 / 3})$ one would have $\lambda=\sqrt{2}$. As estimated for two hcp metals [13], $b^{3} \approx 1.42 v_{W S}$ for $\mathrm{Mg}$ and $1.24 v_{W S}$ for Zn. Hence, we take $\lambda=1.33 \pm 0.09 \approx 4 / 3$. This embraces all of the values quoted above.

In an ensemble of loops there are roughly equal amounts of edge and screw dislocation in the crystal, so we have $1 / \kappa=(1-\nu / 2) \pm \nu / 2 \simeq 5 / 6 \pm 1 / 6$. Therefore, as follows from $(21)$

$$
\ln \left(\frac{2.1}{b^{2} \rho\left(T_{m}\right)}\right)=\frac{2(5 / 6 \pm 1 / 6)}{(1.33 \pm 0.09)(1.01 \pm 0.17)}=1.24 \pm 0.33
$$

Hence,

$$
\rho\left(T_{m}\right)=(0.61 \pm 0.20) b^{-2} .
$$

It follows from Eqs. (21)-(23), with $\kappa \lambda=1.6 \pm 0.3$, that to $\sim 20 \%$ accuracy

$$
T_{m}=\frac{G v_{W S}}{4 \pi \ln (z-1)}
$$

We regard Eq. (24) as a new dislocation melting law.

\section{Free energy of the dislocation ensemble}

To calculate the free energy of a dislocation ensemble, Eq. (15), let us rewrite Eqs. (10) and (12), using Eq. (19) with $R=1 / \sqrt{\rho}$, and replace the sums (which start with $L=4 b$, the smallest loop length) by the corresponding integrals:

$$
\begin{gathered}
Z_{1}=\frac{V}{b^{3}} A\left(q, z^{\prime}\right) \int_{4}^{\infty} d x x^{-q-1}\left[\frac{4 b^{2} \rho}{\alpha^{2}}\left(z^{\prime}\right)^{1 / c}\right]^{c x}, \\
\xi(T)=A\left(q, z^{\prime}\right) \int_{4}^{\infty} d x x^{-q}\left[\frac{4 b^{2} \rho}{\alpha^{2}}\left(z^{\prime}\right)^{1 / c}\right]^{c x}, \quad c \equiv \frac{\kappa G b^{3}}{8 \pi k_{B} T} .
\end{gathered}
$$

Here, $\left(4 b^{2} \rho / \alpha^{2}\right)\left(z^{\prime}\right)^{1 / c}=\exp \left\{-8 \pi \sigma_{\text {eff }} / \kappa G b^{2}\right\} \leq 1$, since $\sigma_{\text {eff }} \geq 0$. Integrating Eq. (25) by parts we find

$$
\begin{gathered}
Z_{1}=\frac{V}{q b^{3}}\left(\frac{\kappa G b^{3}}{8 \pi k_{B} T} \ln \left[\frac{4 b^{2} \rho}{\alpha^{2}}\left(z^{\prime}\right)^{1 / c}\right] \cdot \xi(T)+\frac{A\left(q, z^{\prime}\right)}{4^{q}}\left[\frac{4 b^{2} \rho}{\alpha^{2}}\left(z^{\prime}\right)^{1 / c}\right]^{4 c}\right) \\
=\frac{V}{q b^{3}}\left[-\frac{\sigma_{\mathrm{eff}} b}{k_{B} T} \xi(T)+\frac{A\left(q, z^{\prime}\right)}{4^{q}} e^{-4 \sigma_{\mathrm{eff}} b / k_{B} T}\right] .
\end{gathered}
$$

Hence,

$$
F=-k_{B} T e^{\mu / k_{B} T} Z_{1}=\frac{V}{q b^{3}}\left(\sigma_{\text {eff }} \rho b^{3}-\frac{A\left(q, z^{\prime}\right) e^{\mu / k_{B} T}}{4^{q}} k_{B} T e^{-4 \sigma_{\mathrm{eff}} b / k_{B} T}\right)
$$


where we have replaced $\left(\kappa G b^{3} / 8 \pi\right) \ln \left(\alpha^{2} / 4 b^{2} \rho\right)-k_{B} T \ln z^{\prime}$ by $b \sigma_{\text {eff }}$, in view of (1),(19), and used Eq. (18).

The second term on the right-hand side of Eq. (28) takes its largest value at $T=T_{m}$, where $\sigma_{\text {eff }}=0$. To estimate its contribution to the free energy, consider the case of $\mathrm{Cu}$ discussed in more detail below. In this case, to estimate $A\left(q, z^{\prime}\right) \exp \left\{\mu\left(T_{m}\right) / k_{B} T_{m}\right\} / 4^{q}$, we use Eqs. (12),(18), and replace the sum by an integral:

$$
\frac{A\left(q, z^{\prime}\right) e^{\mu\left(T_{m}\right) / k_{B} T_{m}}}{4^{q}}=\frac{A\left(q, z^{\prime}\right)}{4^{q}} \frac{b^{2} \rho\left(T_{m}\right)}{\xi\left(T_{m}\right)}=\frac{b^{2} \rho\left(T_{m}\right)}{4^{q} \int_{4}^{\infty} d x x^{-q}}=\frac{b^{2} \rho\left(T_{m}\right)(q-1)}{4} .
$$

As discussed in Section 2, the value of $q$ may be expected between 3/2 (Brownian loops) and $\approx 7 / 4$ (self-avoiding loops). With $b^{2} \rho\left(T_{m}\right)$ given in Eq. (23), we therefore obtain $A\left(q, z^{\prime}\right) \exp \left\{\mu\left(T_{m}\right) / k_{B} T_{m}\right\} / 4^{q}=0.095 \pm 0.037 \approx 0.1$.

Hence, the contribution of the second term to $q F / V$ would be $\simeq-0.7 \mathrm{meV}^{-3}$. As seen in Fig. 2, this contribution is negligibly small. In fact, the second zero of $F$ for $T=T_{m}$ and $A\left(q, z^{\prime}\right) \exp \left\{\mu\left(T_{m}\right) / k_{B} T_{m}\right\} / 4^{q}=0.1$ occurs at $b^{2} \rho=0.61$, which is within $\sim 5 \%$ of the value of 0.64 (the second zero of $F$ at $T=T_{m}$ with $F / V$ given in (29)), and within uncertainties in the values of $b^{2} \rho\left(T_{m}\right)$ in Eq. (23).

Thus, we have derived the dislocation free energy density, and it is given approximately by

$$
\frac{q F(\rho)}{V} \simeq \sigma_{\text {eff }} \rho=-\left(\frac{\kappa G}{8 \pi} \ln \left(\frac{4 b^{2} \rho}{\alpha^{2}}\right)+\frac{k_{B} T}{b^{3}} \ln (z-1)\right) b^{2} \rho .
$$

This form for the free energy density was previously suggested but not derived by Cotterill [15]. It was later put on a firm theoretical basis by Yamamoto and Izuyama [17]. It also is a fundamental ingredient in the recently developed theory of dislocation-mediated phase transitions of vortex-line lattices in high- $T_{c}$ superconductors [18.

In Fig. 2 we plot $q F(\rho) / V$ from (29) for $\mathrm{Cu}$ for three different temperatures: $T<T_{m}$, $T=T_{m}$ and $T>T_{m}$. We take $\kappa=6 / 5, \alpha=2.9, G=47.7 \mathrm{GPa}$ [28], $T_{m}=1356$ $\mathrm{K}$, and $b=2.55 \AA$ 倩. A first-order phase transition, that is melting, takes place when the second zero of $F(\rho)$ occurs at the critical dislocation density, $\rho\left(T_{m}\right)$. This is a transition from a perfect crystalline solid to a highly dislocated solid, not a liquid. In fact, our theory describes dislocations, which do not exist in liquids. If a dislocation is viewed as a disclination dipole [29], the dislocated solid may in turn undergo a KosterlitzThouless-like transition [30] to a phase of free disclinations, i.e., a liquid. This dislocated solid may then be viewed as the three-dimensional analog of an intermediate hexatic phase, between a solid and a liquid, in the Halperin-Nelson theory of two-dimensional melting [31]. The clarification of this point needs further investigation, to be undertaken elsewhere. Patashinskii et al. [11] also identified melting as a transition from a perfect crystalline solid to a highly dislocated solid, and Nelson and Toner 32 found residual bond-orientational order in a three-dimensional solid with an equilibrium concentration of unbound dislocation loops, which is analogous to that in the two-dimensional hexatic phase.

Note that it is not possible to increase the dislocation density progressively from zero to $\rho\left(T_{m}\right)$ at a temperature lower than $T_{m}$ (e.g., by deformation) because of the high 
energy barrier at the maximum of $q F(\rho) / V$. Hence, the dislocation density, as a function of temperature, is

$$
\rho(T)=\left[\begin{array}{cc}
0, & T<T_{m} \\
\rho\left(T_{m}\right), & T=T_{m} .
\end{array}\right.
$$

In fact, it can be shown that (30) is the only physical solution of (12) written as a "gap" equation:

$$
e^{-\mu / k_{B} T} b^{2} \rho(T)=A\left(q, z^{\prime}\right) \sum_{n=4}^{\infty} \frac{\left(z^{\prime}\right)^{n}}{n^{q}}\left(\frac{4 b^{2} \rho(T)}{\alpha^{2}}\right)^{\kappa G b^{3} n / 8 \pi k_{B} T} .
$$

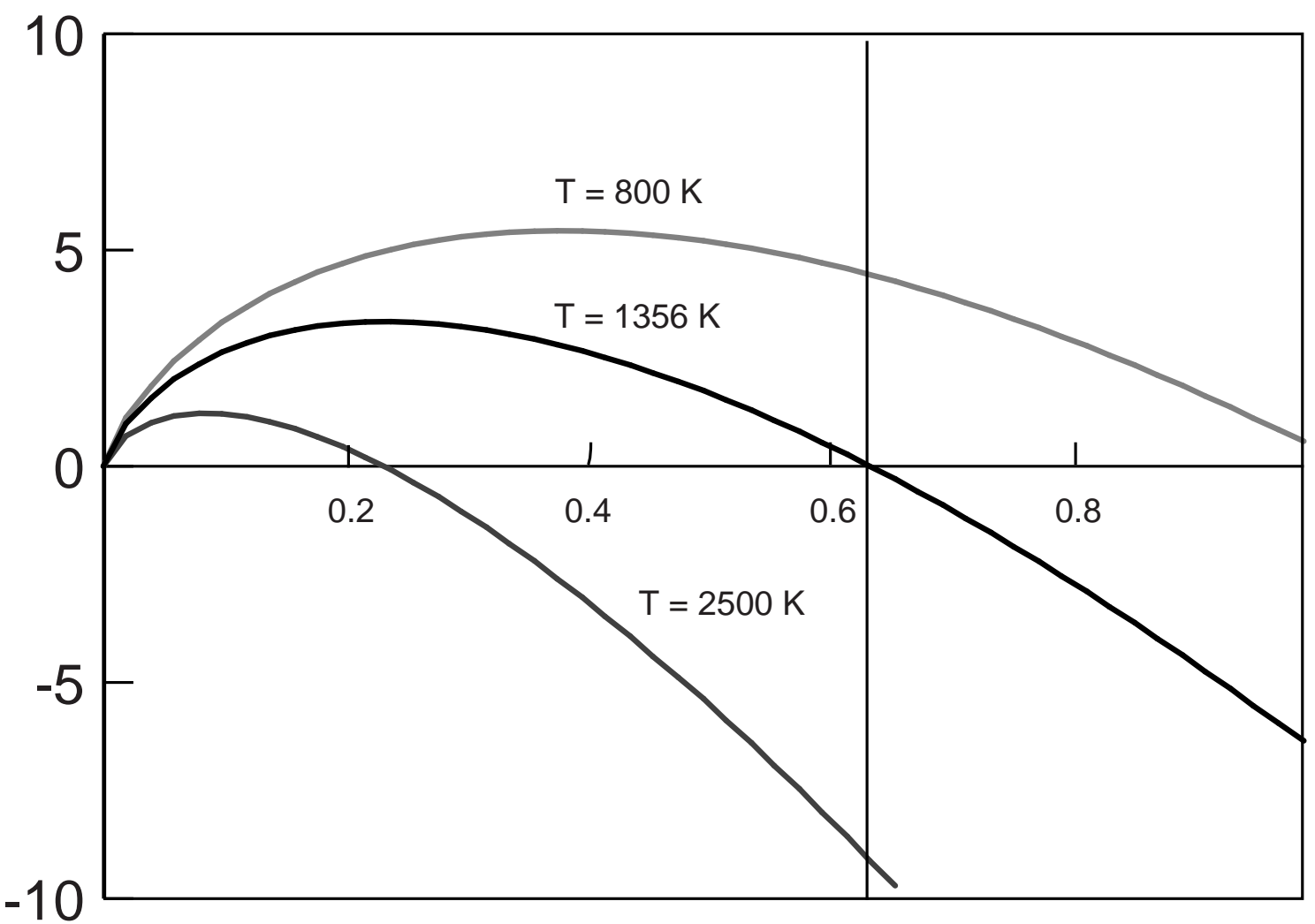

Fig. 2. $q F(\rho) / V$ for $\mathrm{Cu}$ at three different temperatures, in units of $\mathrm{meV}^{-3}$. The vertical line denotes the critical dislocation density value of $0.64 b^{-2}$.

\section{Latent heat of fusion}

For the ensemble of strings on a lattice considered in Section 2, the internal energy and pressure are

$$
U=-\left(\frac{\partial \ln Z}{\partial \beta}\right)_{e^{\mu / k_{B} T}, V}=-\frac{\bar{N}}{Z_{1}} \frac{\partial Z_{1}}{\partial \beta}=e^{\mu / k_{B} T} \frac{V \sigma}{b^{2}} \xi(T)
$$




$$
P=k_{B} T\left(\frac{\partial \ln Z}{\partial V}\right)_{T, \mu}=k_{B} T \frac{\bar{N}}{Z_{1}} \frac{\partial Z_{1}}{\partial V}=e^{\mu / k_{B} T} \frac{k_{B} T}{b^{3}} \bar{\xi}(T) .
$$

Hence, the enthalpy is

$$
H=U+P V=\frac{V}{b^{3}} e^{\mu / k_{B} T}\left[\sigma b \xi(T)+k_{B} T \bar{\xi}(T)\right] .
$$

The latent heat of fusion is the enthalpy difference:

$$
L_{m} \equiv H\left(T_{m}\right)-H(0)
$$

In our case, $H(0)=0$, which follows directly from (30)-(33) and Eq. (18). Using $\sigma_{\text {eff }}\left(T_{m}\right)=$ 0 and the melting condition $k_{B} T_{m}=\sigma b / \ln (z-1)$, we obtain

$$
L_{m}=\frac{V}{b^{3}} e^{\mu\left(T_{m}\right) / k_{B} T_{m}} k_{B} T_{m}\left[\ln (z-1) \xi\left(T_{m}\right)+\bar{\xi}\left(T_{m}\right)\right] .
$$

To obtain the latent heat per mole, the quantity tabulated in the literature, one has to multiply the expression (35) by the ratio of the number of atoms per mole, $N_{A}$, to the total number of atoms in the volume $V$, which is equal to $V / v_{W S}$. Replacing $N_{A} k_{B}$ by the gas constant $R$, and using $b^{3}=\lambda v_{W S}$, we obtain

$$
L_{m}=\frac{e^{\mu\left(T_{m}\right) / k_{B} T_{m}}}{\lambda} \xi\left(T_{m}\right) R T_{m} \ln (z-1)\left[1+\frac{1}{\ln (z-1)} \frac{\bar{\xi}\left(T_{m}\right)}{\xi\left(T_{m}\right)}\right] .
$$

To estimate the ratio $\bar{\xi}\left(T_{m}\right) / \xi\left(T_{m}\right)$, we replace the sums in Eqs. (12),(13) by the corresponding integrals:

$$
\frac{\bar{\xi}\left(T_{m}\right)}{\xi\left(T_{m}\right)}=\frac{\int_{L / b=4}^{\infty} d(L / b)(L / b)^{-q-1}}{\int_{L / b=4}^{\infty} d(L / b)(L / b)^{-q}}=\frac{q-1}{4 q} .
$$

With $3 / 2 \leq q \leq 7 / 4$, as discussed in Section $2,0.083 \leq(q-1) / 4 q \leq 0.107$, i.e.,

$$
\frac{\bar{\xi}\left(T_{m}\right)}{\xi\left(T_{m}\right)}=0.095 \pm 0.012 .
$$

Therefore, the contribution of the second bracketed term on the right-hand side of Eq. (36) (corresponding to the work contribution to enthalpy) is $0.04-0.06(6 \leq z \leq 12)$. We expect, therefore, that neglecting the second bracketed term on the right-hand side of Eq. (36) will introduce an error not larger than $\sim 6 \%$. Hence, with accuracy of $\sim 94 \%$ we have the following formula for the latent heats of the elements:

$$
L_{m}=\frac{1}{\lambda} b^{2} \rho\left(T_{m}\right) R T_{m} \ln (z-1)
$$

where we have replaced $e^{\mu\left(T_{m}\right) / k_{B} T_{m}} \xi\left(T_{m}\right)$ by $b^{2} \rho\left(T_{m}\right)$, in view of (18). The proportionality of latent heat of fusion to the critical concentration of defects (multiplied by the core energy) has been noted previously by Cotterill [15]. 
In Fig. 3 we plot the values of $b^{2} \rho\left(T_{m}\right)$ extracted from the experimental data on latent heats for 75 elements. For this analysis, the values of both $T_{m}$ and $L_{m}$ are mostly taken from [33]. For Be, Hf, Sc, Sr, Y, the lanthanides Dy, Ce, Er, Gd, Ho, La, Nd, Sm, Tb, Yb, and the actinides Am, Cm, Th, we disregard their high- $T$ bcc phases which exist only in the very vicinity of melting. (The intermediate $\mathrm{hcp} \rightarrow$ fcc phase transition for $\mathrm{Yb}$, dhcp $\rightarrow$ fcc for Am, Ce and La, and fcc $\rightarrow$ hcp for Sr, as well as hcp $\rightarrow$ fcc for Co, do not change coordination number.) The crystal structure chosen for the evaluation of $\mathrm{Ca}, \mathrm{Co}, \mathrm{Mn}, \mathrm{N}$, $\mathrm{Np}, \mathrm{O}, \mathrm{Sm}, \mathrm{Ti}, \mathrm{Tl}, \mathrm{U}$ and $\mathrm{Zr}$ corresponds to the phase from which melting occurs. The data on both $T_{m}$ and $L_{m}$ for $\mathrm{H}, \mathrm{N}, \mathrm{O}, \mathrm{Pa}$ and Rn are taken from [34]. The data on both $T_{m}$ and $L_{m}$ for Am and $\mathrm{Cm}$, and on $L_{m}$ for $\mathrm{Ar}, \mathrm{Kr}, \mathrm{Ne}$ and Xe are taken from [35]. The data on $L_{m}$ for the lanthanides are taken from [36]. The following values of $\lambda$ are used: 1 for sc, 1.3 for bcc, 1.41 for fcc, 1.24 for Zn, 1.42 for $\mathrm{Mg}$, and 1.33 for all other elements.

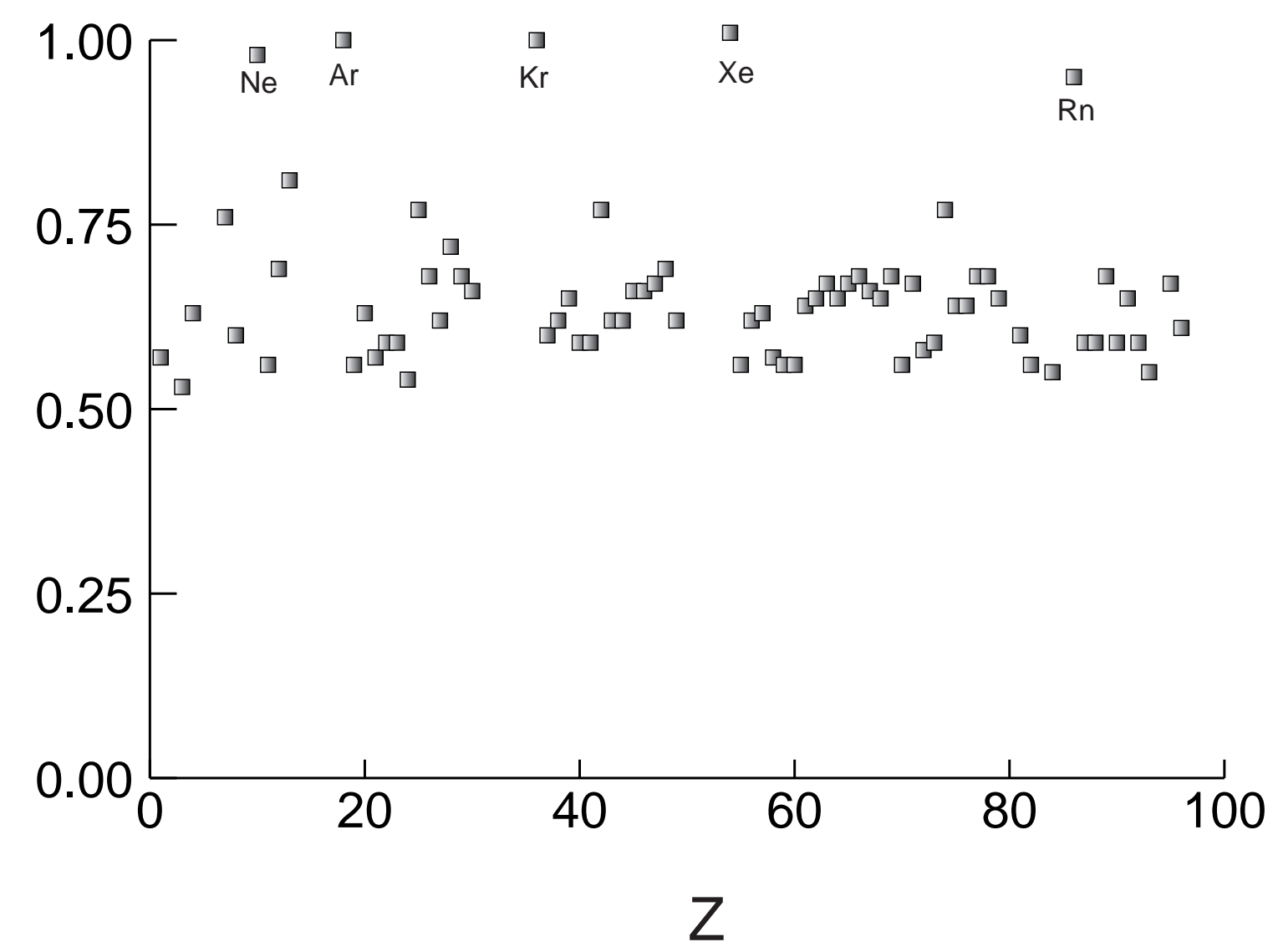

Fig. 3. Critical dislocation density as extracted from the experimental data on latent heat of fusion for 75 elements. 
For all these elements we find

$$
\rho\left(T_{m}\right)=(0.66 \pm 0.11) b^{-2}
$$

where the error is the root-mean-square deviation. This value is in good agreement with that obtained from the melting temperatures alone, Eq. (23).

Note that the possible inaccuracy in the value of $\lambda$ for the hcp, dhcp and bct elements used in this analysis, on the order of $\sim 7 \%$, may slightly increase uncertainty in the value of $\rho\left(T_{m}\right)$ in Eqs. (23) and (40).

We do not have a reasonable explanation for the anomalously high values of $\rho\left(T_{m}\right)$ for the noble gases. If the noble gases are excluded from the analysis, then $\rho\left(T_{m}\right)$ turns out to be $(0.63 \pm 0.06) b^{-2}$ for the remaining 70 elements. Note also that for deuterium (D), which is not included in Fig. 3, with the data on $T_{m}$ and $L_{m}$ from [35, we obtain $b^{2} \rho\left(T_{m}\right)=0.70$.

The uncertainty-weighted average of the values of $\rho\left(T_{m}\right)$ given in Eqs. (23) and (40) is

$$
\rho\left(T_{m}\right)=(0.64 \pm 0.14) b^{-2},
$$

which we take as our result for the critical dislocation density at melt.

\section{Volume change at melt}

As an independent consistency check on the relations (23) and (40), we determine the critical dislocation density using the formula [13, 37]

$$
\varepsilon \equiv \frac{\triangle V}{V}=\frac{\lambda}{2 \pi} \frac{G}{B}\left(\gamma_{G}-\frac{1}{3}\right) b^{2} \rho\left(T_{m}\right)
$$

where $\triangle V$ is the difference between the liquid and solid specific volumes at melt, $G$ and $B$ are the shear and bulk moduli, respectively, and $\gamma_{G}$ is the Grüneisen constant. Here, $\varepsilon$ is identified with the dilation of the lattice as the reaction of the crystal to the sudden

proliferation of dislocations. In Table 1 we show the values of $b^{2} \rho\left(T_{m}\right)$ calculated for 32 elements for which we could find zero-pressure data on $\gamma_{G}$ and $\varepsilon$. The experimental values of $\varepsilon$ are mostly taken from ref. [35], and those of $G, B$ and $\gamma_{G}$ from [28]. For Ar, $\mathrm{Kr}, \mathrm{Ne}$ and Xe, the values of $G$ and $B$ are taken from [38], and those of $\gamma_{G}$ from [39]. 


\begin{tabular}{|c||c|c|c|c||c||c|}
\hline element & $B, \mathrm{GPa}$ & $G, \mathrm{GPa}$ & $\gamma_{G}$ & $\varepsilon$ & $b^{2} \rho\left(T_{m}\right)$ from Eq. $(42)$ & $b^{2} \rho\left(T_{m}\right)$ from Eq. $(39)$ \\
\hline $\mathrm{Ag}$ & 103 & 29.8 & 2.40 & 0.052 & 0.39 & 0.67 \\
$\mathrm{Al}$ & 76.0 & 26.1 & 2.19 & 0.064 & 0.44 & 0.81 \\
$\mathrm{Ar}$ & 1.83 & 0.75 & 2.59 & 0.144 & 0.69 & 1.00 \\
$\mathrm{Au}$ & 173 & 28.0 & 2.99 & 0.055 & 0.57 & 0.65 \\
$\mathrm{Be}$ & 111 & 151 & 1.11 & 0.115 & 0.51 & 0.63 \\
$\mathrm{Ca}$ & 16.7 & 7.4 & 1.15 & 0.048 & 0.64 & 0.63 \\
$\mathrm{Cs}$ & 2.01 & 0.65 & 1.41 & 0.026 & 0.36 & 0.56 \\
$\mathrm{Cu}$ & 137 & 47.7 & 2.02 & 0.046 & 0.35 & 0.68 \\
$\mathrm{Eu}$ & 17.0 & 7.53 & 1.39 & 0.048 & 0.50 & 0.67 \\
$\mathrm{Gd}$ & 37.8 & 21.6 & 0.63 & 0.021 & 0.59 & 0.65 \\
$\mathrm{Ho}$ & 40.8 & 26.3 & 1.18 & 0.075 & 0.65 & 0.66 \\
$\mathrm{In}$ & 42.0 & 4.78 & 2.43 & 0.025 & 0.49 & 0.62 \\
$\mathrm{~K}$ & 3.3 & 0.9 & 1.29 & 0.025 & 0.46 & 0.56 \\
$\mathrm{Kr}$ & 2.04 & 0.85 & 2.64 & 0.151 & 0.70 & 1.00 \\
$\mathrm{Li}$ & 12.1 & 3.85 & 0.92 & 0.016 & 0.41 & 0.53 \\
$\mathrm{Lu}$ & 47.6 & 27.2 & 1.06 & 0.036 & 0.41 & 0.67 \\
$\mathrm{Na}$ & 6.74 & 1.98 & 1.19 & 0.027 & 0.52 & 0.56 \\
$\mathrm{Nb}$ & 171 & 37.6 & 1.77 & 0.029 & 0.44 & 0.59 \\
$\mathrm{Nd}$ & 32.9 & 17.4 & 0.57 & 0.009 & 0.34 & 0.56 \\
$\mathrm{Ne}$ & 0.88 & 0.40 & 2.79 & 0.156 & 0.62 & 0.98 \\
$\mathrm{Ni}$ & 183 & 85.8 & 1.93 & 0.063 & 0.37 & 0.72 \\
$\mathrm{~Pb}$ & 44.7 & 8.6 & 2.74 & 0.037 & 0.36 & 0.56 \\
$\mathrm{Pd}$ & 193 & 48.0 & 2.56 & 0.059 & 0.47 & 0.66 \\
$\mathrm{Pt}$ & 283 & 63.7 & 2.87 & 0.066 & 0.51 & 0.68 \\
$\mathrm{Rb}$ & 2.3 & 0.63 & 0.99 & 0.026 & 0.70 & 0.60 \\
$\mathrm{Ta}$ & 193 & 69.0 & 1.74 & 0.052 & 0.50 & 0.59 \\
$\mathrm{~Tb}$ & 38.7 & 22.1 & 0.74 & 0.032 & 0.65 & 0.67 \\
$\mathrm{Tl}$ & 35.7 & 5.4 & 2.10 & 0.033 & 0.60 & 0.60 \\
$\mathrm{Tm}$ & 46.2 & 29.1 & 1.43 & 0.069 & 0.47 & 0.68 \\
$\mathrm{~W}$ & 310 & 160 & 1.67 & 0.090 & 0.63 & 0.77 \\
$\mathrm{Xe}$ & 2.1 & 1.0 & 2.56 & 0.130 & 0.54 & 0.56 \\
$\mathrm{Yb}$ & 14.9 & 8.06 & 1.04 & 0.036 & 0.44 & \\
& & & & & & 01 \\
\hline
\end{tabular}

Table 1. Values of $b^{2} \rho\left(T_{m}\right)$ from experimental data on volume change at melt for 32 elements. For comparison, we also show values of $b^{2} \rho\left(T_{m}\right)$ extracted for the same elements from the data on latent heats.

For all 32 elements in Table 1 we find

$$
\rho\left(T_{m}\right)=(0.51 \pm 0.11) b^{-2},
$$

where the error is the root-mean-square deviation. This is somewhat lower than but still in agreement with both Eqs. (23) and (40) taking into account uncertainties associated with the three values.

For comparison, we show in the last column of Table 1 the values of $b^{2} \rho\left(T_{m}\right)$ extracted for the same elements from the data on $L_{m}$. It is seen that the agreement between two sets of the values of $b^{2} \rho\left(T_{m}\right)$ is reasonably good, except for $\mathrm{Ag}, \mathrm{Al}, \mathrm{Cs}, \mathrm{Cu}, \mathrm{Ni}, \mathrm{Pb}$ and $\mathrm{Xe}$, for which the difference in both values of $b^{2} \rho\left(T_{m}\right)$ is on the order of $\sim 50-60 \%$, Lu, $\mathrm{Ne}$ and $\mathrm{Nd}$ for which the difference is $\sim 45 \%$, and $\mathrm{Ar}, \mathrm{Kr}, \mathrm{Pd}$ and $\mathrm{Tm}$, for which it is $\sim 35 \%$. For all other elements, the difference does not exceed $\sim 30 \%$. 
Note that the contribution of the volume change at melt, $\varepsilon$, to the latent heat of fusion is proportional to $\varepsilon^{2} \ll 1$ [13, 37], and is therefore negligibly small compared to the right-hand side of Eq. (39).

\section{Concluding remarks}

Our theory of dislocation-mediated melting was developed in the approximation that dislocations are non-interacting. This approximation is good only in the vicinity of melt where the dislocation density is very high and the otherwise long-range interactions are sufficiently screened [27]. The statistical mechanics of non-interacting dislocations on a lattice yields simple, accurate relations between the dislocation density at melt and both the melting temperature and latent heat of fusion, despite the indeterminacy of the parameter $q$ that takes into account the possible non-Brownian nature of the dislocation network. The values of $\rho\left(T_{m}\right)$, as determined from an extensive analysis of $T_{m}$ and $L_{m}$ data, are remarkably consistent: $(0.61 \pm 0.20) b^{-2}$ and $(0.66 \pm 0.11) b^{-2}$, respectively. The uncertainty-weighted average of these values is $\rho\left(T_{m}\right)=(0.64 \pm 0.14) b^{-2}$, which we take as our result for the dislocation density at melt. Poirier and Price [13 analyzed 14 elements and found $\rho\left(T_{m}\right) v_{W S} / b=0.48 \pm 0.12$. Using $v_{W S}=b^{3} / \lambda$ with $\lambda \approx 4 / 3$, their result corresponds to $\rho\left(T_{m}\right)=(0.64 \pm 0.16) b^{-2}$, which is in excellent agreement with ours. Kierfeld and Vinokur [18] modelled dislocation-mediated phase transitions of a vortex-line lattice and found $\rho\left(T_{m}\right) \approx 0.6 b^{-2}$. Vachaspati's 40 study of topological defect formation gave $a^{2} \rho\left(T_{m}\right) \approx 0.88$ for a simple cubic lattice. This translates into $\rho\left(T_{m}\right) \approx 0.66 b^{-2}$ for bcc lattices $(a=2 / \sqrt{3} b)$ and $\rho\left(T_{m}\right) \approx 0.44 b^{-2}$ for fcc lattices $(a=\sqrt{2} b)$, which are consistent with our result. In agreement with Vachaspati, Kibble [41] found $a^{2} \rho\left(T_{m}\right) \approx 0.89$ for a simple cubic lattice.

Although our main results do not depend on the precise value of $q$, there is a particular value of $q$ at which the relations (29) and (39) become exact: $q=1$. In this limit, as seen in (12), $\xi\left(T_{m}\right) \rightarrow \infty$, so that Eq. (39) becomes exact in view of (36). Requiring finite internal energy in this limit leads, via (31), to $\exp \left\{\mu\left(T_{m}\right) / k_{B} T_{m}\right\} \rightarrow 0\left(\mu\left(T_{m}\right) \rightarrow-\infty\right)$, and therefore, Eq. (29) becomes exact, since the second term on the right-hand side of (28) disappears. In fact, the study of cosmological networks of string loops in 3 dimensions by Magueijo, Sandvik and Steer [42 results in a scale-invariant loop distribution of the form of Eqs. (9),(10) with $q+1<5 / 2$ : 43 $1.9<q+1<2.1$, or $44 q+1=2.03$ (plus error bars), and so in this study $q \approx 1$. Thus, it is quite possible that linear defects which correspond to two apparently distinct physical phenomena, namely cosmic strings and crystal dislocations, are of a very similar statistical-mechanical nature.

The average total dislocation length per Wigner-Seitz cell at melt is $\rho\left(T_{m}\right) v_{W S}=$ $b \rho\left(T_{m}\right) / \lambda \approx b / 2$, since $\lambda \approx 4 / 3$. Since a Wigner-Seitz cell contains $z$ links, each of length $b / 2$, it follows that, on average, one of $z$ links in each Wigner-Seitz cell is covered by a dislocation. Since each such a link is shared between two atoms, on average, half of the atoms are within a dislocation core at melt.

If we use $\rho\left(T_{m}\right)=0.64 b^{-2}$, then to $\sim 20 \%$ accuracy the melting temperatures and 
latent heats are given by

$$
\begin{aligned}
& k_{B} T_{m}=\frac{G v_{W S}}{4 \pi \ln (z-1)}, \\
& L_{m}=\frac{\ln (z-1)}{2} R T_{m} .
\end{aligned}
$$

The accuracy of these relations depends critically on the factor of $\ln (z-1)$, which is characteristic of a theory based on line-like degrees of freedom.

\section{Acknowledgements}

We thank T. Goldman for valuable discussions during the preparation of this work. One of us (L.B.) wishes to thank J. Magueijo and D.A. Steer for very useful correspondence.

\section{References}

[1] W. Shockley, in L'Etat Solide, Proceedings of Neuvienne-Consail de Physique, Brussels, 1952, Ed. R. Stoops, (Solvay Institut de Physique, Brussels, Belgium)

[2] W.L. Bragg, in Proc. Symp. on Internal Stresses, (Institute of Metals, London, 1947), p. 221

[3] R.M.J. Cotterill and M. Doyama, Phys. Rev. 145 (1966) 465

[4] C. Mott, Proc. Roy. Soc. A 215 (1952) 1

[5] R.M.J. Cotterill, Phys. Rev. Lett. 42 (1979) 1541

[6] W. Janke, Int. J. Theor. Phys. 29 (1990) 1251

[7] R.K. Crawford, Bul. Am. Phys. Soc. 24 (1979) 385

R.M.J. Cotterill and J.K. Kristensen, Phil. Mag. 36 (1977) 453

[8] S. Mizushima, J. Phys. Soc. Japan 15 (1960) 70

[9] A. Ookawa, J. Phys. Soc. Japan 15 (1960) 2191

[10] M. Siol, Z. Phys. 164 (1961) 93

D. Kuhlmann-Wilsdorf, Phys. Rev. 140 (1965) A1595

S.F. Edwards and M. Warner, Phil. Mag. A 40 (1979) 257

S.P. Obukhov, Zh. Eksp. Teor. Fiz. 83 (1982) 1978

[11] A.Z. Patashinskii and B.I. Shumilo, Zh. Eksp. Teor. Fiz. 89 (1985) 315

A.Z. Patashinskii and L.D. Son, Zh. Eksp. Teor. Fiz. 103 (1993) 1087

[12] E. Copeland, D. Haws, S. Holbraad and R. Rivers, Physica A 179 (1991) 507 
[13] J.P. Poirier and G.D. Price, Phys. Earth Planet. Inter. 69 (1992) 153

[14] H. Kleinert, Gauge Fields in Condensed Matter, (World Scientific, Singapore, 1989), Vol. II, and references therein

[15] R.M.J. Cotterill, J. Cryst. Growth 48 (1980) 582

[16] B.I. Halperin, Statistical mechanics of topological defects, in Physics of Defects, Eds. R. Balian, M. Kleman and J.P. Poirier, (North Holland, Amsterdam, 1981) J.P. Poirier, Geophys. J. Roy. Astr. Soc. 85 (1986) 315

[17] T. Yamamoto and T. Izuyama, J. Phys. Soc. Japan 57 (1988) 3742

[18] J. Kierfeld and V. Vinokur, Dislocations and the critical endpoint of the melting line of vortex line lattices, cond-mat/9909190

[19] L. Burakovsky and D.L. Preston, Los Alamos preprint LA-UR-99-4171 condmat/0003494], to appear in Solid State Comm.

[20] F.W. Wiegel, Introduction to Path-Integral Methods in Physics and Polymer Science, (World Scientific, Singapore, 1986)

[21] M.B. Green, J.H. Schwarz and E. Witten, Superstring Theory, (Cambridge University Press, Cambridge, 1987), Vol. 1

[22] E. Copeland, D. Haws, S. Holbraad and R. Rivers, Physica A 158 (1989) 460, Nucl. Phys. B 319 (1989) 687

[23] R. Brandenberger and C. Vafa, Nucl. Phys. B 316 (1989) 391

[24] P. Salomonson and B.S. Skagerstam, Nucl. Phys. B 268 (1986) 349, Physica A 158 (1989) 499

[25] D.A. Lowe and L. Thorlacius, Phys. Rev. D 51 (1995) 665

[26] J.P. Hirth and J. Lothe, Theory of Dislocations, 2nd ed., (Krieger Publishing, Malabar, FL, 1992)

[27] G.F. Sarafanov, Phys. Solid State 39 (1997) 1403

[28] M.W. Guinan and D.J. Steinberg, J. Phys. Chem. Solids 35 (1974) 1501

[29] N.D. Mermin, Rev. Mod. Phys. 51 (1979) 591

N.K. Gilra, Cryst. Lattice Defects 8 (1979) 59

K.J. Strandburg, Rev. Mod. Phys. 60 (1988) 161

[30] J.M. Kosterlitz and D.J. Thouless, J. Phys. C 6 (1973) 1181, in Progress in Low Temperature Physics, Vol. VII-B, Ed. D.F. Brewer, (North-Holland, Amsterdam, 1978), p. 373. The 3D Kosterlitz-Thouless-like transition is discussed in C. Giannessi, J. Phys. Cond. Mat. 3 (1991) 1649, and N.K. Kultanov and Yu.E. Lozovik, Solid State Comm. 88 (1993) 645, Physica Scripta 56 (1997) 129 
[31] D.R. Nelson and B.I. Halperin, Phys. Rev. Lett. 41 (1978) 121, Phys. Rev. B 19 (1979) 2456

[32] D.R. Nelson and J. Toner, Phys. Rev. B 24 (1981) 363

[33] K.A. Gschneidner, Jr., in Solid State Physics, Advances in Research and Applications, Eds. F. Seitz and D. Turnbull, (Academic Press, New York, 1965), Vol. 16, p. 275

[34] J. Emsley, The Elements, (Clarendon Press, Oxford, 1989)

[35] E. Yu. Tonkov, High Pressure Phase Transformations, (Gordon and Breach, Philadelphia, 1992)

[36] Handbook of the Physicochemical Properties of the Elements, Ed. G.V. Samsonov, (IFI/Plenum, New York, 1968)

[37] T. Ninomiya, J. Phys. Soc. Japan 44 (1978) 263

[38] P. Korpiun and E. Lüscher, in Rare Gas Solids, Eds. M.L. Klein and J.A. Venables, (Academic Press, London, 1977), Vol. II, p. 741

[39] V.S. Vorob'ev, High Temp. 34 (1996) 197

[40] T. Vachaspati, Phys. Rev. D 44 (1991) 3723

[41] T.W.B. Kibble, Phys. Lett. B 166 (1986) 311

[42] J. Magueijo, H. Sandvik and D.A. Steer, Phys. Rev. D 60 (1999) 103514

[43] D.A. Steer, private communication

[44] J. Magueijo, private communication 